\title{
SMART AND NOVEL IMPROVEMENTS IN CONTINUOUS CASTING ADD TO BIG COST CUTTING BENEFITS*
}

\author{
Thomas Schanz $z^{1}$ \\ Marcus Feldmann ${ }^{2}$ \\ Phoebe Härtner ${ }^{3}$ \\ Pierpaolo Rivetti ${ }^{4}$ \\ Dino Kabosch ${ }^{5}$ \\ Giovanni Michelon ${ }^{6}$
}

\begin{abstract}
Current steel market conditions require small investments only to lower operational cost. SMS Concast has developed new products which optimize continuous casting process cost for long product steel production. This paper describes a selection of three products. All three systems are patented (patent pending for CONDRIVE). CONSAFE, a non-radioactive mould level measurement system for reliable mould level measuring on open stream casting CCM's; CONSTIR-MWS, an improved control software for electromagnetic stirrers which lowers energy cost by $35 \%$; CONDRIVE, a brand new oscillation drive system which provides the features of a hydraulic oscillator, however with a cost efficient electric drive.

Keywords: Continous casting; Mould level measuring; Radioactivity (absence of); Electromagnetic stirring; Modulation; Mould oscillation; Oscillation drive; Cost savings; Green technology; Ecofriendly.
\end{abstract}

1 SMS Meer Ltda., Barueri, Brazil

2 Head of Product Management, BSc in Mechanical Engineering; MASc of Applied Science in Business Administration, SMS Concast AG, Zurich, Switzerland

3 Sales \& Marketing Manager, MSc Management, Technologies \& Economics (ETH Zurich), SMS Concast AG, Zurich, Switzerland

4 Vice President - Asia Pacific, Sales \& Marketing, SMS Concast AG, Zurich, Switzerland

5 Development \& Product Design Engineer, MSc in Engineering FH, SMS Concast AG, Zurich, Switzerland

6 Head of Development \& Product Design E\&A, Degree in Automation Control Engineering, SMS Meer S.p.A., Tarcento, Italy. 


\section{CONSAFE}

\section{Introduction and benefits}

Radioactive mould level measuring systems for continuous casting machines are still common. However and despite its advantages [1], the use of radioactive devices adds several layers of cost including licensing, working health and safety, operating practices and disposal of radioactive sources after use. SMS Concast has developed CONSAFE, a safe, radiation-free, reliable and less expensive alternative to conventional mould level measuring systems. Especially when a plant needs to replace its radioactive devices or the detectors, then the return on investment (ROI) becomes very short.

\section{Working principle}

CONSAFE uses a patented array of four thermocouples which is attached to the mould tube (Figure 1). The tips of the thermocouples are inserted into holes in the mould tube. This ensures optimal thermal contact and grants for best dynamic response. As the steel level rises in the mould tube, temperature changes due to heat transfer are sensed by the sensor array. An advanced algorithm evaluates the thermocouple signals to determine the level of the liquid steel inside the mould. The raw thermocouple signals are converted into highly noise-immune $4-20 \mathrm{~mA}$ temperature data which is then applied to a thermal model in the controller, producing a representation of the actual steel level in the mould tube.

The arrangement of a CONSAFE installation is shown in Figure 2. At the start of the cast, CONSAFE is capable of recognizing the raising steel level within the mould before the steel reaches the control range of the thermocouples. This allows the PID in the CONSAFE controller to take control of the strand at a very early stage. The steel level within the mould tube does not overshoot the set-point. This steel level pre-view function provides for a safe and reliable auto start.

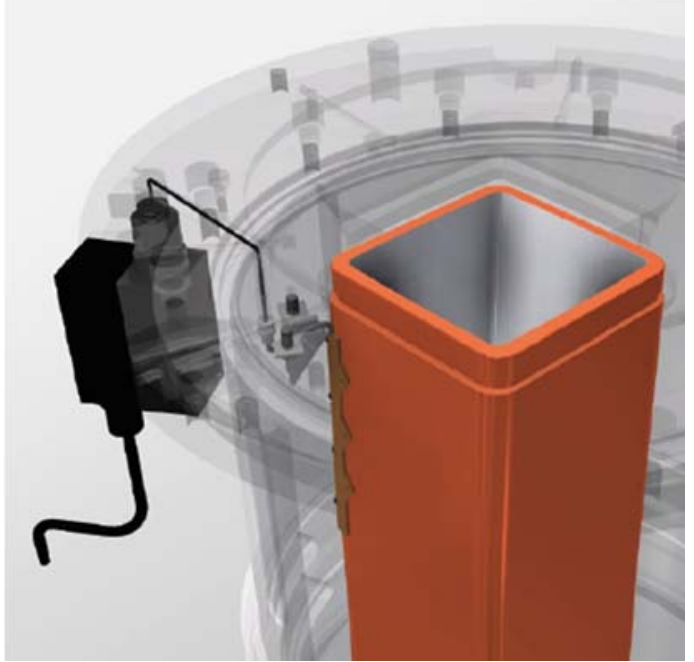

Figure 1. Scheme of sensor array mounted on a mould tube with cabling from wet side to dry side.

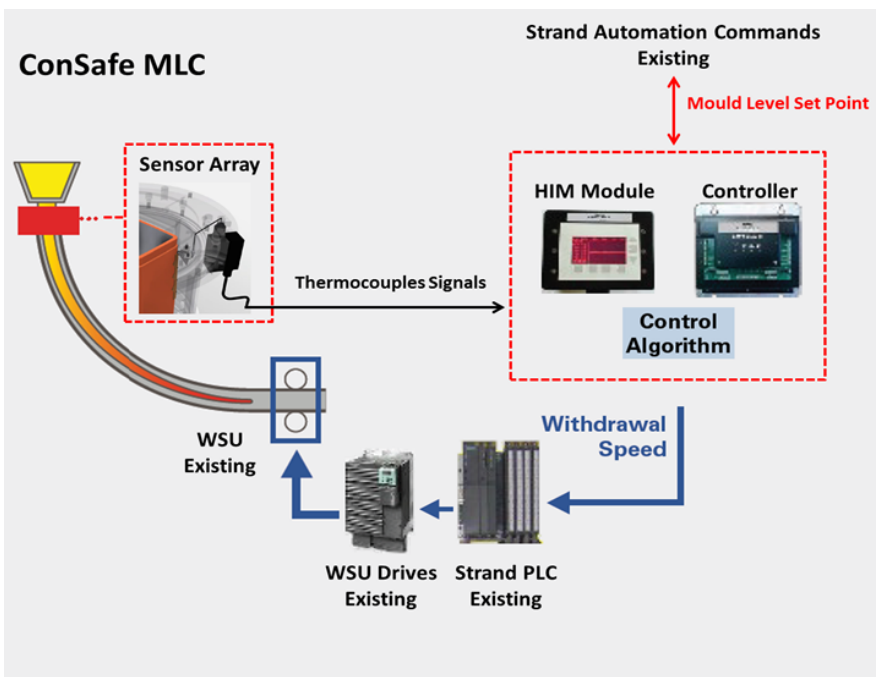

Figure 2. CONSAFE system components and layout. 


\section{Application in the plant}

CONSAFE works independent os the casting section size, steel grade or casting speed, however exclusively for open casting only. The sensor can be fitted into practically every open stream caster. Attaching a sensor array is a fairly simple and quick operation. The water jacket is not affected. The lower top plate of the mould cartridge requires a threaded hole to safely guide out the cable. No re-calibration is required after the initial system calibration during commissioning. The system is maintenance-free. Even in the unlikely event of two thermocouple failures, it would still sense and hold a level to operate the strand until the end of the cast.

Learning to operate the system is easily accomplished by the intuitive design of the interface screen. Online operational data across all strands is available on the same display. The controller is capable of storing operational data for one year of production, giving extensive diagnostics and data trending capabilities. Communication is via Ethernet. Field network connection is adaptable to Profibus, Profinet etc.

\section{Operating results}

The red graph in Figure 3 shows the stability of the mould level controlled by CONSAFE. This is the result of what an advanced algorithm record of the thermocouples The first installation of CONSAFE happened in 2001 under the name of its inventor who was offering the system mainly in the North American market. As of the year 2015, SMS Concast bought the intellectual property of this smart product and brings it to the entire world. CONSAFE comes with an impressive track record of ten long product mills in the USA and three mills elsewhere.

Customers [2] who are nowadays using CONSAFE are happy with it firstly because of its outstanding reliability: The systems have been working continuously for several years and need practically no attention to keep them operational. Secondly, customers appreciate that they got the many issues about radioactivity solved. All cost bound to radioactivity which usually occur in several organizational departments of one plant are cut with immediate effect. The third fact why steel plants decide for CONSAFE is that the any chance of radioactive decontamination is also immediately stopped. Working health and safety concerns in this respect are solved.

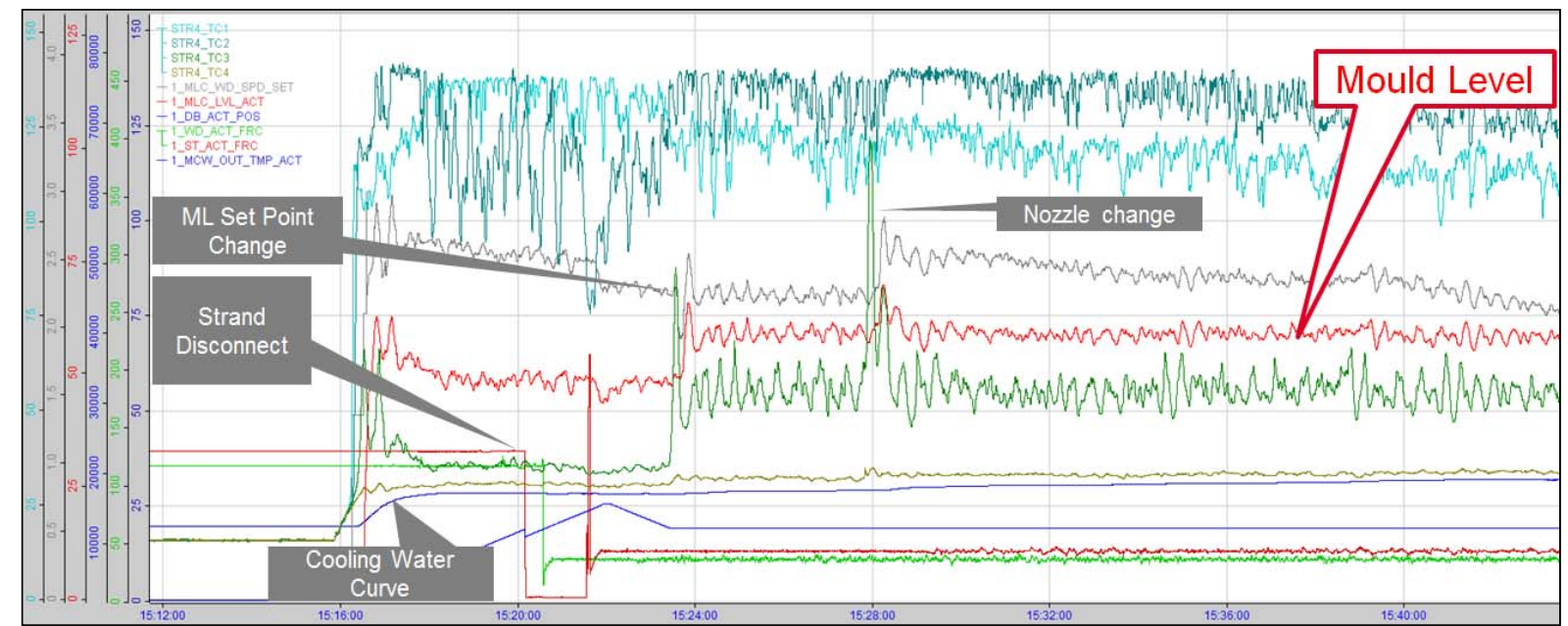

Figure 3 shows the mould level, which is hardly affected by events such as a nozzle change. 


\section{CONSTIR-MWS}

\section{Introduction and benefits}

CONSTIR-MWS is part of SMS Concast's electromagnetic stirrer product family, and MWS stands for Modulated Wave Stirring. The new product is software which modulates the current and frequency of the stirrer. Operated by CONSTIR-MWS a stirrer will consume $35 \%$ less electric power than conventional systems. Such power savings generate a ROI of less than 1 year in many cases.

The lower energy input also reduces the angular velocity of the steel meniscus which is an advantage for the casting operation itself, but it also provides improvement to carbon segregation and potentially also for porosity $[3,4]$.

\section{Working principle}

The CONSTIR-MWS technology intensifies the heat and mass transfer processes of the liquid steel inside a continuously casting strand. The conventional sinusoidal magnetic field (Figure 4) is replaced by a multi-frequency magnetic field, generated with amplitude and frequency modulation (Figure 5). The modulated wave carries less energy; hence the power savings are achieved. The modulation changes the melt circulation pattern in the vicinity of the solidification front and provides positive metallurgical effects for shell growth.

A proprietary mathematical model is used which calculates how the conventional sinusoidal current input for an EMS is to be modulated in its amplitude (AM) and frequency (FM). The calculation variables are steel grade- and section-specific and operational inputs.

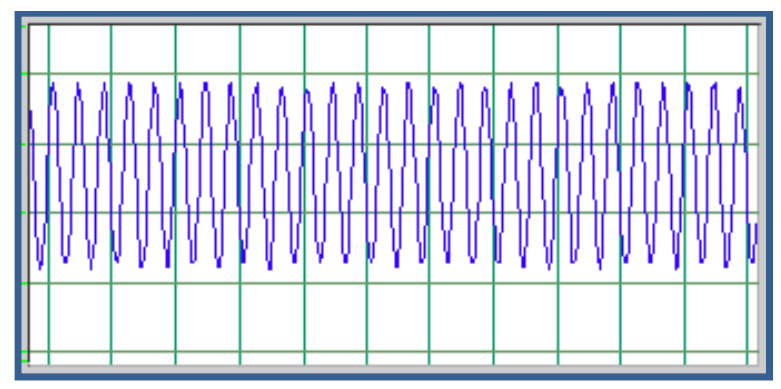

Figure 4 . Conventional sinusoidal magnetic field.

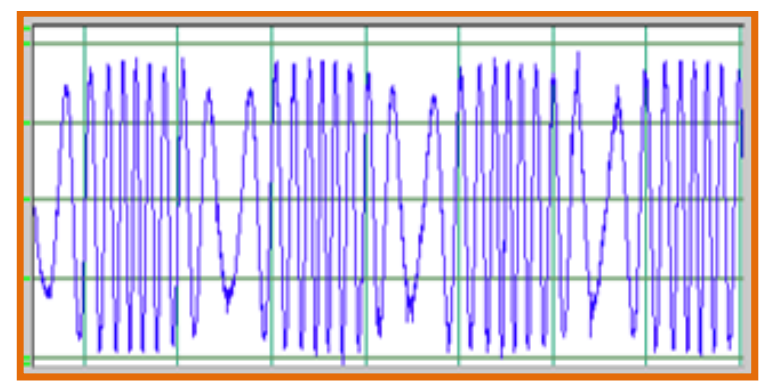

Figure 5 . CONSTIR-MWS modulated magnetic field.

\section{Application in the plant}

The software can be implemented on most CCM's, even those equipped with existing mould stirring units. Figure 6 shows the layout of an industrial implementation. In both cases, the frequency converter is controlled from the CCM control room with the metallurgical recipe system. A CONSTIR-MWS controller is integrated into the overall system. The modulation control of the EMS system is realized by a special algorithm installed in the new CONSTIR-MWS controller which generates the amplitude and frequency modulation set points. The set points are then provided to the frequency converters, which generate the modulation. The original EMS system now performs a fast and reliable AM/FM operation. 


\section{CONSTIR-MWS stirring}

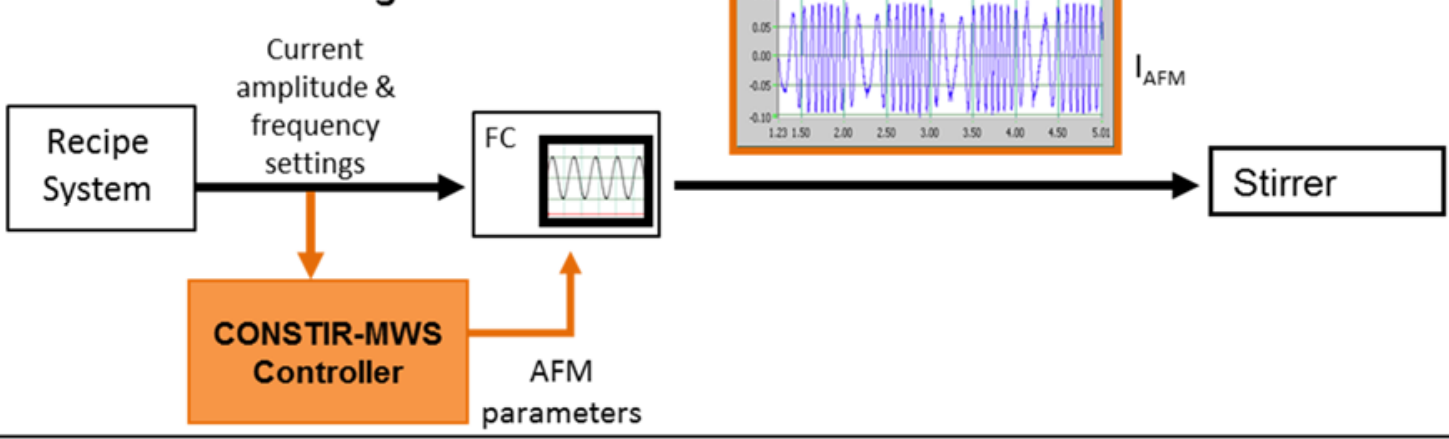

Figure 6: Industrial implementation of CONSTIR-MWS

\section{Operating results}

Several industrial implementations exist and many trials have been performed to gain clear evidence on the superiority of this technology.

\section{Energy savings}

The average energy saving achieved on seven CONSTIR-MWS installations averaged at $35.4 \%$ (Table 1). Such power consumption savings are quite an achievement, especially if considering that the stirrers are often the largest energy consumers on a CCM. In some countries, plants receive governmental advantages if they can present energy consumption savings in this range. The energy savings are easily measured during commissioning. All energy savings figures presented within Table 1 were evaluated as the difference between CONSTIR-MWS switched off and on.

\begin{tabular}{|c|c|c|c|c|}
\hline Plant & Country & Type & Stirrers & Power Savings \\
\hline 1 & Russia & 6 strand billet & M-EMS & $34.4 \%$ \\
\hline 2 & Turkey & 8 strand billet & M-EMS & $36.1 \%$ \\
\hline 3 & Poland & 3 strands billet & M-EMS \& F-EMS & $33.3 \%$ \\
\hline 4 & Italy & 3 strand bloom & M-EMS \& F-EMS & $35.5 \%$ \\
\hline 5 & England & 6 strand bloom & M-EMS & $37.2 \%$ \\
\hline 6 & Switzerland & 4 strand billet & M-EMS \& F-EMS & $41.4 \%$ \\
\hline 7 & USA & 3 strand bloom & M-EMS & $30.0 \%$ \\
\hline \multicolumn{4}{|c|}{ Average measured electrical energy savings: } & $35.4 \%$ \\
\hline
\end{tabular}

Table 1: Electrical energy savings with CONSTIR-MWS on seven CCM's

\section{Centerline porosity}

Figure 7 shows an impressive statistical result of central porosity improvement achieved in a steel plant using M-EMS and F-EMS for casting of peritectic steel with analysis $\quad 0.18-0.2 \% \mathrm{C}, \quad 0.15-0.25 \% \mathrm{Si}, \quad 0.85-1.05 \% \mathrm{Mn}, \quad<0.15 \% \mathrm{Cr}, \quad<0.15 \% \mathrm{Ni}$, $\mathrm{Mo}<\mathrm{Cu}<0.18 \%$ ) in a $200 \mathrm{~mm}$ square section. It can be seen that the size of the pores is reduced if using CONSTIR-MWS. 


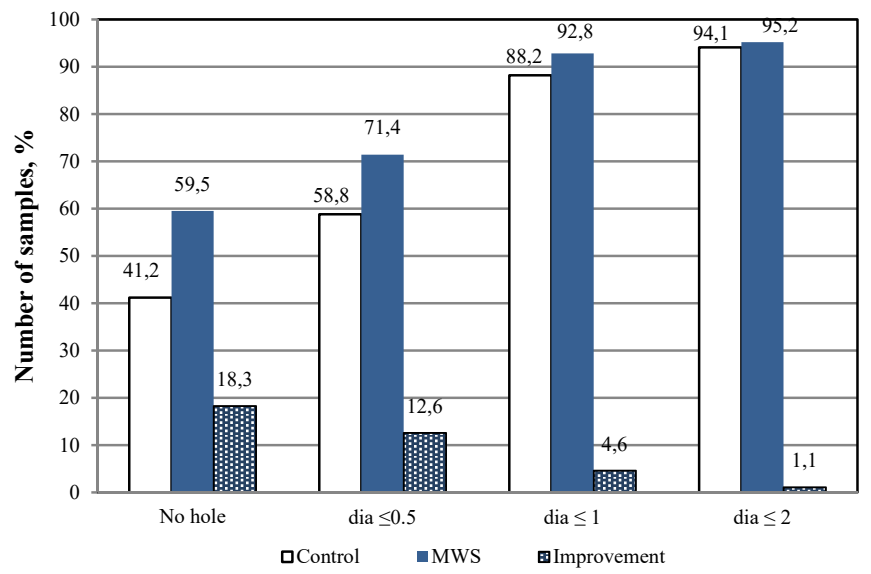

Figure 7 Central porosity of peritectic steel in $200 \mathrm{~mm}$ square billets

\section{Carbon segregation, white band and equiaxed zone}

Carbon segregation and its standard deviation generally decreased in the statistical analyses. The carbon coefficients of all strand sample slices produced with CONSTIR-MWS ranged in a smaller band (from best to worst) as compared to the band of all strand sample slices stirred in the conventional way. The positive impact of CONSTIR-MWS on impurity segregation can be explained by the resonance condition effects and with the decreasing of the average angular velocity of the melt. This action may lead to a reduction of the white band, together with reduction of the dendritic structure. There are few methods to increase the size of the equiaxed zone but in some cases, the equiaxed zone is positively affected by applying CONSTIRMWS.

\section{CONDRIVE}

\section{Introduction and benefits}

One main part in a continuous casting machine is the mould oscillation, crucial for flawless mould lubrication and final surface quality. Oscillation parameters, such as stroke and frequency need to be adapted to the steel grades and casting modes (e.g. open or closed casting, casting speed and other). Very frequently hydraulic drives are used in the mould oscillation in order to generate mould oscillation curves adaptable during operation. This is very helpful in order to optimize the oscillation according to the steel grade and current casting conditions. On the other hand hydraulic drives are more costly by nature due to the whole hydraulic installation needed. Another simple solution for mould oscillation is often an electrical eccentric drive. The latter is advantageous cost wise but does not allow for oscillation curve changes during operation. The CONDRIVE combines the simple design of the electrical eccentric oscillation with the flexibility of a hydraulic cylinder (see comparison Table 2). 


\begin{tabular}{|l|l|l|l|l|}
\hline \multicolumn{2}{|c|}{ Electrical oscillation } & \multicolumn{1}{|c|}{ Hydraulic oscillation } & \multicolumn{2}{c|}{ CONDRIVE } \\
\hline+ & Simple design & + & $\begin{array}{l}\text { Stroke and frequency are } \\
\text { changeable during casting } \\
+\end{array}$ & Low maintenance costs \\
+ & - & $\begin{array}{l}\text { Stroke and frequency are } \\
\text { changeable during casting } \\
\text { Cheap solution } \\
\text { piping needed } \\
\text { Manual manipulation to } \\
\text { adjust the stroke }\end{array}$ & - & $\begin{array}{l}\text { High maintenance cost } \\
\text { Low maintenance costs } \\
\text { Very good value for money } \\
\text { Just electrical power and cooling } \\
\text { water is needed }\end{array}$ \\
\hline
\end{tabular}

Table 2 Comparison of different oscillation drive systems

Through a part-turn movement of an eccentric shaft the sinusoidal movement at the mould is generated. This concept is patented by SMS Concast AG for oscillation in steel production. In general, the CONDRIVE can replace any single hydraulic drive in billet casters (see Figure 8). Further applications will be checked in future.

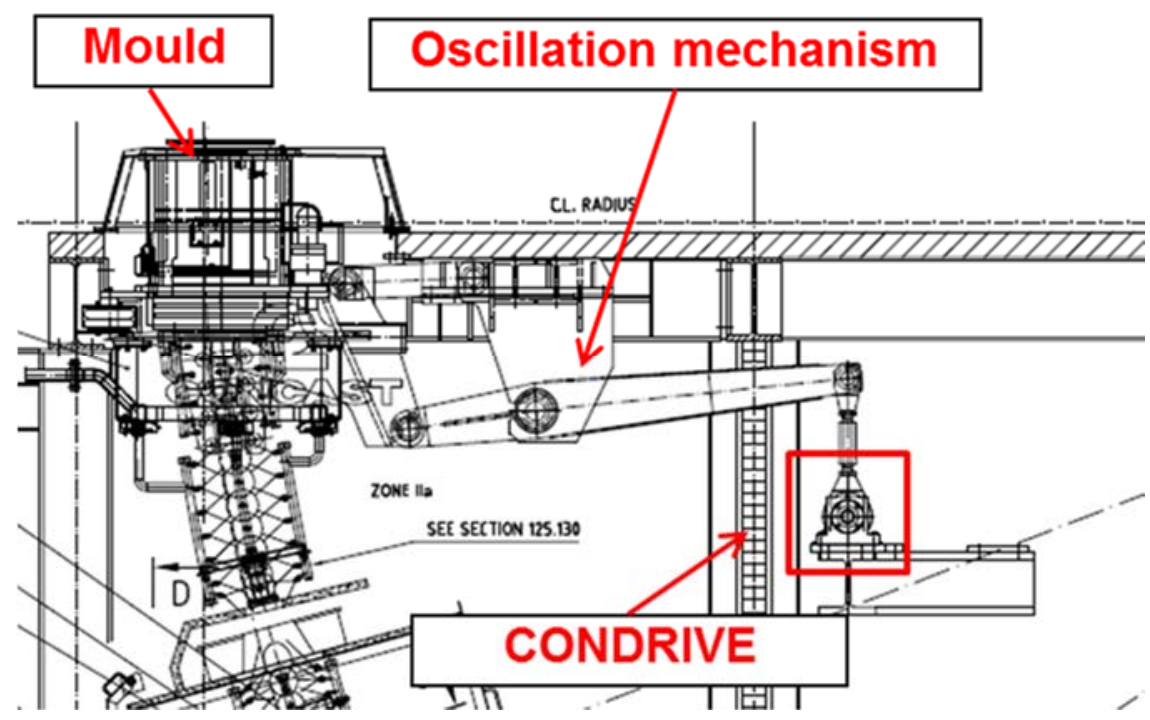

Figure 8: CONDRIVE Oscillation drive implemented in a continuous casting machine (here with short lever oscillation)

\section{Working Principle}

The sinusoidal oscillation with a stroke $s$ can be generated by a part-turn movement of $\pm \alpha$ (see Figure 9). The stroke and/or frequency can be adjusted independently by the software at any time during casting by changing the maximum angle $\pm \alpha$ and/or the turning speed. 


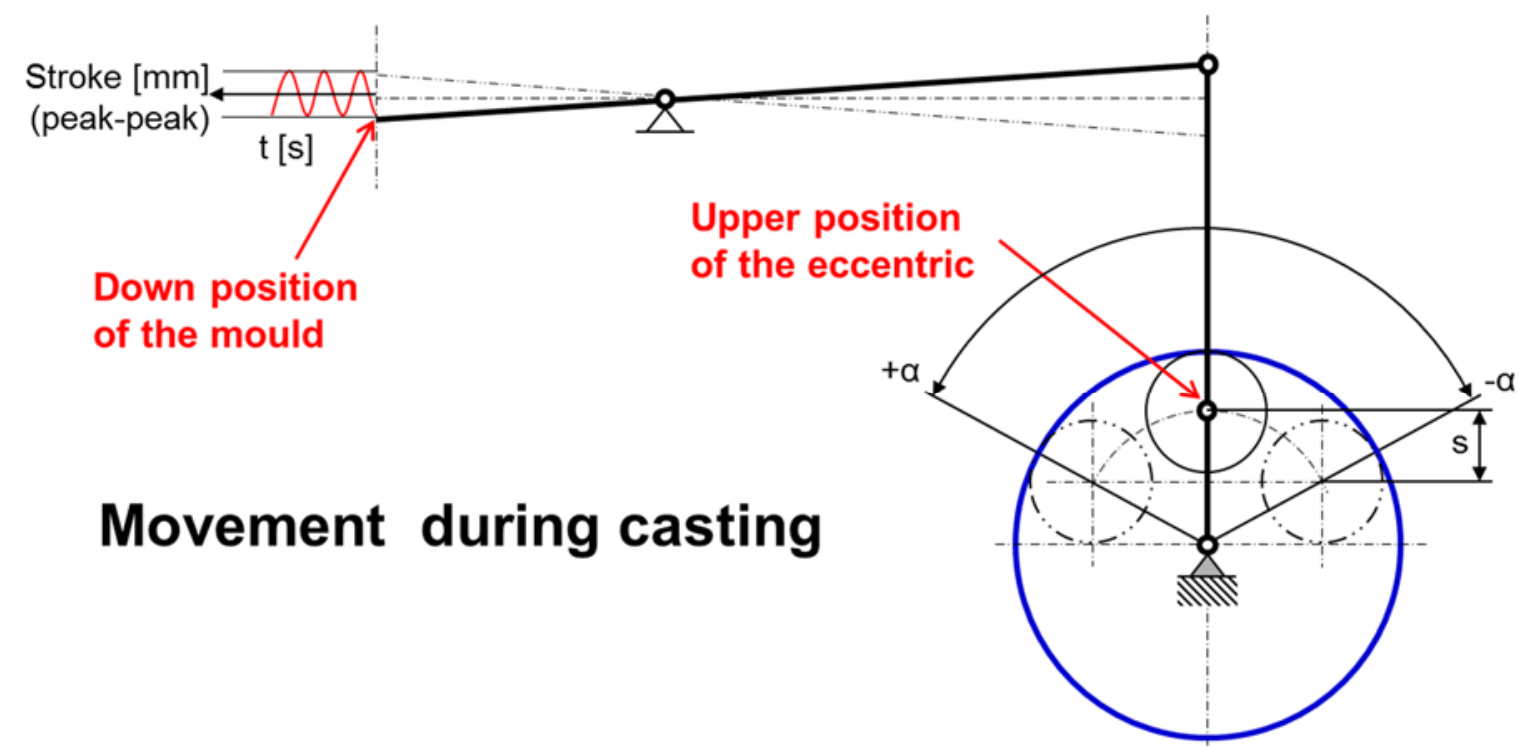

Figure 9: CONDRIVE working principle

\section{Application in the plant}

The CONDRIVE can generate any parameter combination of a sinusoidal oscillation for a stroke (peak to peak) up to $18 \mathrm{~mm}$ and a frequency of up to $5 \mathrm{~Hz}\left(300 \mathrm{~min}^{-1}\right)$. The only restriction is the maximal acceleration at the mould of $3.5 \mathrm{~m} / \mathrm{s}^{2}$ to protect the oscillation mechanism.

To run the CONDRIVE the following is needed:

\section{- Electrical power \\ - Cooling water \\ - Servo drive with electrical components}

The size of the CONDRIVE is approximately $600 \mathrm{~mm} \times 450 \mathrm{~mm} \times 660 \mathrm{~mm}$ (length $\mathrm{x}$ width $\mathrm{x}$ height). It combines the following features:

- Online Stroke and Frequency adjustment during casting (no manual manipulation)

- Freedom to choose various oscillation curves (e.g. asymmetric sinus, saw tooth)

- Online mould friction measurement is possible

- No hydraulic power unit or piping is required

- No gearbox is used and therefor the only wear occurs in the bearing

- The expected maintenance interval is three to five years beside the central greasing interval

- The price and maintenance costs are lower than the hydraulic oscillation with the same functionality

These features allow for a very efficient and flexible mould oscillation, reducing capital as well as operational expenses.

\section{Operating results}

CONDRIVE is still a brand new product which is currently shortly ahead of market introduction. 


\section{CONCLUSION}

The three technologies described are examples for innovative products by SMS Concast. Each one has a thoroughly sustainable positive effect on the operating cost of a steel plant. These products can be implemented with a comparably low CAPEX, and ROI time is short. Summarized, the technologies show following advantages:

1. CONSTIR-MWS: Reduced stirrer power consumption and improved metallurgical results

-power consumption savings by up to $30 \%$ and more

-Reduced centreline porosity (see Figure 7) and decreased carbon segregation

2. CONSAFE: Avoidance of radioactivity for open casting mould level measurement and safe operations

-Minimal implementation cost (almost neglectable)

-All cost related to radioactivity (like licences, trainings etc) are omitted

3. CONDRIVE: Reduced CAPEX and OPEX in mould oscillation whilst keeping or even improving the oscillation performance compared to hydraulic and electric oscillation drives.

$-50 \%$ less CAPEX compared to hydraulic oscillation drives

-no more OPEX related to hydraulics for the oscillation

\section{REFERENCES}

1. Fabrizioli M., Michelon G. Michelon G. Dal Corso F., Borsato E., Fries S., Ney G., Nagy R., New radiometric sensor for mould level measurement allowing separate detection of meniscus and powder thickness, Proc. METEC 2015.

2. Feldmann M., Gregord J., Michelon G., Caskey R., "CONSAFE - Innovative Mould Level Measuring for Open Stream Casting”, AISTech 2016 Conference, Pittsburgh, USA, May 16-18, 2016.

3. M. Feldmann, L. Neri, S. Lesin, A. Kapusta, M. Khavkin, B. Tilman, "Current and Frequency Modulation: New Way to Improve Electromagnetic Stirring", 2nd ESTAD, European Steel Technology and Application, Düsseldorf, June 18, 2015.

4. Feldmann M., Michelon G., Neri L., Novak H., Klipa N., Lesin S., Kapusta A., Khavkin M., Tilman B., "CONSTIR-MWS - Boosting of Electromagnetic Stirring Results", AISTech 2016 Conference, Pittsburgh, USA, May 16-18, 2016.

Contact: Markus Feldmann, Head of Product Management at SMS Concast AG, Zurich, Switzerland, markus.feldmann@sms-concast.ch, www.sms-concast.ch 